\title{
Marco legal contra el tabaquismo: Referente obligado para la lucha antitabáquica
}

\author{
Legal Frame against Tobacco-Addiction: \\ an Anti-Tobacco Campaign Reference:
}

Dra. Angélica Ramírez Elías

\section{Resumen}

El tabaquismo es la principal causa mundial de enfermedades y muertes evitables. Muchas de éstas son, además, prematuras. En México esta adicción se ha convertido en un problema de salud pública. La última Encuesta Nacional de Tabaquismo reporta que, la epidemia de tabaquismo se ha incrementado en los países industrializados y en vías de desarrollo; la brecha entre el consumo de hombres y mujeres es muy similar, y el consumo se inicia a una edad cada vez más temprana, en los (primeros años de la adolescencia). La Organización Mundial de Salud (OMS) refiere que el hábito tabáquico mata casi seis millones de personas cada año, de los cuales cinco millones son usuarios y ex usuarios y más de 600,000 son no fumadores expuestos al humo de segunda mano; y es que a menos de que se tome acción, la cuota anual de muertes pudiera aumentar a más de 8 millones para el 2030; y casi el 80\% de un millón de millones de fumadores viven en países de bajos y medianos ingresos. El Convenio Marco Contra el Tabaquismo(CMCT) auspiciado por la OMS es el primer tratado internacional sanitario en materia de tabaquismo. México fue el primer país en América Latina en refrendar el CMCT tras reconocer la magnitud del problema y sus complicaciones tanto en adultos como en menores de edad. El objetivo de este acuerdo es proporcionar un marco para identificar medidas de lucha antitabáquica que habrán de adoptarse mediante el compromiso de las partes para reducir el consumo de tabaco y proteger así a las generaciones presentes y futuras contra las consecuencias sanitarias, sociales, ambientales y económicas del consumo y de la exposición al humo del tabaco.

Profesor de carrera asociado C ENEO-UNAM

Responsable del Macro proyecto formación de recursos en salud para la prevención de conductas adictivas Folio MP6-20

Correspondencia: arelias1999@yahoo.com

RECIBIDO 5 DE DICIEMBRE 2010

ENVIADO 7 DE FEBRERO 2011

ACEPTADO 28 DE FEBRERO 2011 


\section{Enfermería Universitaria}

\section{InNOVACión para La PRÁCTiCa}

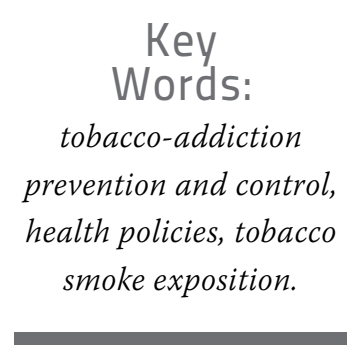

Abstract

Tobacco-addiction is one of the principal causes of preventable illnesses and premature deaths in the world. In Mexico, tobacco-addiction has become a more serious public health problem. Data from the last National Survey on TobaccoAddiction suggest that its prevalence in developing countries has increased, its male-female consumption pattern has become more similar, and that it now starts at younger ages - the first years of adolescence. The World Health Organization (WHO) stated that tobacco now kills nearly six million people each year of whom more than 5 million are users and ex users and more than 600000 are nonsmokers exposed to second-hand smoke; that unless urgent action is taken, the annual death toll could rise to more than eight million by 2030; and that nearly $80 \%$ of the world's one billion smokers live in low-and middle-income countries. An Agreement Frame against Tobacco Addiction supported by the WHO is one of the international sanitary treaties against tobacco addiction in which Mexico has acknowledged the magnitude of the problem and its complications for both adults and children. The objective of this agreement is to provide a frame to identify measures to fight against tobacco-addiction in order to protect present and future generations from tobacco smoke inhalation and its sanitary, social, environmental, and economical consequences.

\section{INTRODUCCIÓN \\ Un problema global}

La evidencia científica generada en los últimos años demuestra que el tabaco es uno de los productos más nocivos para la salud y que la nicotina es una sustancia que induce adicción, por lo que el combate a su consumo se ha convertido para los sistemas de salud del mundo en una prioridad.

Hoy en día, más de 1000 millones de personas fuman tabaco en todo el mundo - de las cuales aproximadamente la cuarta parte son adultos-, y su consumo mata a más de cinco millones cada año. Si las tendencias actuales se mantienen, para 2030 el tabaco será la causa de mortalidad de aproximadamente ocho millones de personas cada año. Para finales de este siglo, el tabaco podría haber matado a 1000 millones de personas. Se estima que más de las tres cuartas partes de esas defunciones se pro- ducirán en países de ingresos bajos y medianos. ${ }^{1}$

El consumo de tabaco constituye un factor de riesgo de seis de las ocho principales causas de mortalidad en el mundo. Fumar tabaco se asocia con cáncer de pulmón, laringe, riñón, vejiga, estómago, colon, cavidad oral y esófago, así como leucemia, bronquitis crónica, enfermedad pulmonar obstructiva crónica, cardiopatía isquémica, infarto, aborto y parto prematuro, defectos de nacimiento e infertilidad, entre otras enfermedades. Todas causas de sufrimiento prevenible y pérdida de años de vida productiva en las personas. Los costos económicos del consumo de tabaco incluyen salarios perdidos, la reducción de la productividad y el aumento en los costos de la asistencia sanitaria. ${ }^{1-2}$
Con frecuencia, y de forma equivocada, se considera que el consumo de tabaco es una opción exclusivamente personal. Contradice esa percepción el hecho de que la mayoría de los fumadores desea abandonar su hábito cuando adquiere plena conciencia de los efectos en la salud, aunque les resulte difícil dejarlo debido a que la nicotina es muy adictiva. Aunado a los efectos de una industria poderosa a nivel mundial la cual invierte decenas de miles de millones de dólares anualmente en su comercialización, y se sirve de grupos de presión y publicistas muy cualificados para mantener e incrementar el consumo de tabaco. ${ }^{1}$

Se ha demostrado que diversas estrategias reducen el consumo de tabaco. Sin embargo, después de 50 años de que los peligros para la 
salud quedaran demostrados científicamente, y más de 20 años después de que la evidencia confirmara los riesgos que entraña el humo ajeno, son muy pocos los países que han aplicado estrategias eficaces y reconocidas para controlar la epidemia de tabaquismo. En este panorama mundial, se reconoce como un logro sin precedentes el establecimiento por la Organización Mundial de la Salud (oms) del Convenio Marco para el Control del Tabaco (СМCT), que permite a los países suscritos conjuntar políticas, directrices, esfuerzos y recursos para combatirlo en todo el mundo. ${ }^{1-5}$

Los esfuerzos internacionales dirigidos por la OMS condujeron a la entrada en vigor del CMCT la que cuenta con 168 signatarios y más de 150 Partes. Este convenio establece los principios y el contexto para la formulación de políticas, la planificación de intervenciones y la movilización de recursos políticos y financieros para el control del tabaco. La consecución de las metas relacionadas con el control del tabaco requerirá la coordinación entre numerosos organismos gubernamentales, instituciones académicas, asociaciones de profesionales y organizaciones de la sociedad civil en el plano nacional, así como el apoyo coordinado de la cooperación internacional y los organismos de desarrollo.

Las partes en el СмCт de la OMS se han comprometido a proteger la salud de sus poblaciones uniéndose a la lucha contra la epidemia de tabaquismo. Con el fin de ayudar a los países a cumplir la promesa del СMCT de la OMS y convertir ese consenso mundial en una realidad a escala. ${ }^{1-3}$

\section{Desarrollo}

\section{Características del CMCT}

El Convenio Marco para el Control del Tabaco de la Organización Mundial de la Salud (СмСт-OMS) es el primer tratado internacional que reafirma el derecho de todas las personas a tener el más alto nivel de salud y constituye un instrumento

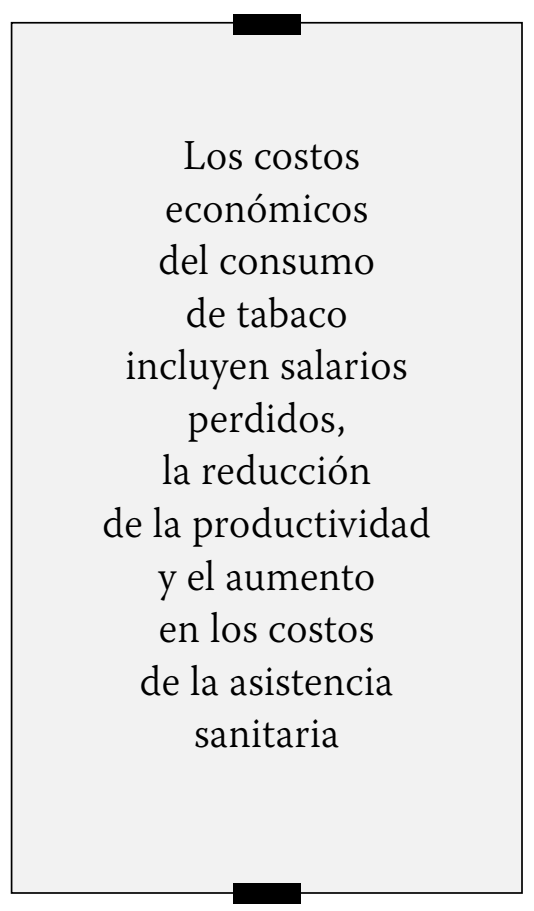

jurídico concebido para reducir la morbimortalidad atribuible al tabaquismo. Sus disposiciones establecen normas internacionales para el control del tabaco en términos de publicidad, promoción y patrocinio; adopción de medidas fiscales - como el precio, empaquetado y etiquetado- el tráfico ilícito y la protección frente al humo de tabaco en el ambiente. Con el fin de proteger a toda la población frente a las consecuencias sanitarias, económicas y sociales atribuibles a esta adicción. En octubre de 2000, los Estados Miembros de la Ops se unieron a otros Estados Miembros de la OMs para empezar las negociaciones sobre el Convenio Marco para el Control del Tabaco (CMCT). Los Estados Miembros de la OMs, reunidos en la Asamblea Mundial de la Salud en mayo de 2003, ratifican un CMCT. ${ }^{4-5}$

El CMCT-OMS entró en vigor el 27 de febrero de 2005 y al mes de mayo de 2006, 129 Estados Miembros de la oms ya hacían parte del mismo. En su preámbulo, éste acuerdo reconoce la relación que existe entre los derechos humanos y el tabaco: ${ }^{3,5-6}$

...Recordando el artículo 12
del Pacto Internacional de De-
rechos Económicos, Sociales
y Culturales, adoptado por la
Asamblea General de las Nacio-
nes Unidas el 16 de diciembre
de 1966 , en el que se declara
que toda persona tiene derecho
al disfrute del más alto nivel
posible de salud física y mental.

El СмСт-OMs incluye acciones específicas para proteger al individuo de la exposición al humo de tabaco ajeno y de informar al público sobre sus riesgos. La obligación central de las partes en relación con la exposición al humo de tabaco ajeno se encuentra en el artículo 8, Protección contra la exposición al humo de tabaco. ${ }^{3-5}$

"Las Partes reconocen que la
ciencia ha demostrado de ma-
nera inequívoca que la exposi-
ción al humo de tabaco es cau-
sa de mortalidad, morbilidad y
discapacidad"

Otras obligaciones establecidas en CMCT-OMS se relacionan con la disponibilidad de información para el público. El artículo 12, Educación, comunicación, formación y concientización del público, recalca la importancia de la educación 
pública acerca de la exposición al humo de tabaco ajeno y requiere que las partes adopten medidas para promover, entre otras cosas:

(a) un amplio acceso a programas integrales y eficaces de educación y concienciación del público sobre los riesgos que acarrean para la salud el consumo de tabaco y la exposición al humo de tabaco, incluidas sus propiedades adictivas;

b) la concientización del público acerca de los riesgos que acarrean para la salud el consumo de tabaco y la exposición al humo de tabaco, así como de los beneficios que reportan el abandono de dicho consumo y los modos de vida sin tabaco... ${ }^{3}$

El derecho internacional y el CMCT-OMS proporcionan una ruta para la acción, los siguientes puntos ofrecen orientación sobre las estrategias centrales:

- el artículo 8 requiere la protección contra la exposición al humo de tabaco ajeno en TODOS lugares públicos cerrados, los medios de transporte público y los lugares de trabajo;

- el artículo 12 requiere un acceso amplio a la educación y a los programas de concientización del público sobre los riesgos para la salud de la exposición al humo de tabaco ajeno;

- la revisión, sanción e implementación de legislación, políticas, planes y prácticas consistentes con el derecho internacional de derechos humanos;

- la protección de todos los ciudadanos sin discriminación y en pie de igualdad ante la ley, consistente con el derecho internacional sobre derechos humanos; $y$

- la accesibilidad y difusión de la información acerca de la exposición al humo de tabaco ajeno consistente con el derecho de toda persona a buscar y recibir información (derecho a la libertad de expresión según el derecho internacional de derechos humanos)..$^{3,1-6}$

Dentro de las Américas, el mandato central de los gobiernos para abordar la exposición al humo de tabaco ajeno es la Resolución CD43. R12 del Consejo Directivo de la Organización Panamericana de la Salud (OPS), la cual, entre otras cosas, insta a los Estados Miembros de la OPS: a que protejan a todos los no fumadores, en particular los niños y las mujeres embarazadas, de la exposición al humo de tabaco en el ambiente mediante la prohibición inmediata del acto de fumar en los edificios del gobierno, establecimientos de asistencia sanitaria e instituciones educativas y mediante la creación, lo antes posible, de entornos sin humo de tabaco en los centros de trabajo y lugares públicos, reconociendo que los ambientes sin humo de tabaco también promueven la cesación del consumo de tabaco y previenen su inicio. ${ }^{3}$

Posiblemente la obligación más importante de acuerdo al derecho internacional de derechos humanos sea la obligación de implementar la legislación, las políticas, los planes y prácticas que permitan garantizar que los derechos humanos estén protegidos verdaderamente en el ámbito nacional. Si esto no ocurre, el derecho internacional se reduce a una afirmación de principios que no se ponen en práctica. Los gobiernos nacionales cuentan con la facultad de reglamentar el uso de tabaco en los lugares públicos así como en los lugares de trabajo y deberían implementar las leyes, políticas, planes y prácticas, guiados por sus obligaciones de derechos humanos, que estipulan que todos estos entornos deben estar 100\% libres del humo del tabaco. En algunas circunstancias, es posible que los entornos exteriores también puedan ajustarse a esta disposición.

Toda la legislación y demás medidas nacionales deberían incluir alguna mención que las vincule con la protección de los derechos humanos pertinentes consagrados en los instrumentos de derechos humanos internacionales e interamericanos. ${ }^{1,3-4}$

\section{El convenio marco para el control del tabaco en México}

México fue el primer país en América Latina en refrendar el Convenio Marco para el Control del Tabaco (CMLA) tras reconocer la magnitud del problema y sus complicaciones tanto en adultos como en menores de edad.

El objetivo del CMLA y nuestro compromiso como país ante el problema de la epidemia del tabaquismo, es proporcionar un marco para identificar medidas de lucha antitabáquica que habrán de adoptarse para reducir el consumo de tabaco y proteger así a las generaciones presentes y futuras contra las consecuencias sanitarias, sociales, ambientales y económicas de su consumo y de la exposición al humo del tabaco. ${ }^{2}$ 
El tabaquismo en México se ubica dentro de las patologías que requieren atención emergente, los datos de la última Encuesta Global de Tabaquismo en Adultos realizada en 2009 y Encuesta 2009 de Tabaquismo en Jóvenes en México, demuestran que existe una fuerte vinculación del tabaquismo con los principales indicadores de morbilidad y mortalidad. Se estima que 122 personas mueren diariamente por causas asociadas al tabaco, se perfila como una enfermedad no transmisible que, de no establecer medidas integrales para su control, rebasará la capacidad sanitaria que se requiere para su atención., ${ }^{2,6-7}$.

El 15.9\% (10.9 millones) de los adultos en México son fumadores actuales, $24.8 \%$ de los hombres (8.1 millones) y $7.8 \%$ (2.8\% millones) de las mujeres. El 48\% de los fumadores actuales son fumadores diarios. El $99 \%$ de los hombres y $96 \%$ de mujeres fuman cigarrillos manufacturados. Entre los fumadores diarios de cigarrillos, en promedio, los hombres fuman 9.7 cigarrillos por día y las mujeres 8.4. La edad de inicio de fumar entre los fumadores diarios de 18-43 años fue de 16.3 años para los hombres y 16.8 años para las mujeres. ${ }^{6-7}$

Entre los 68.8 millones de personas mayores de 15 años en México, $17.8 \%$ (12.2 millones) permite que se fume en su casa y $6.4 \%$ están expuestos al humo de tabaco ajeno en su casa diariamente y aproximadamente 11.9 millones (17.3\%) están expuestos al humo de tabaco ajeno (HTA) en el interior de sus hogares. ${ }^{7}$

El 19.7\% (3.8 millones) de los que laboran en lugares cerrados están expuestos al HTA, incluido $17.7 \%$ (2.6 millones) que son no fumadores. Para estos trabajadores,
$73.6 \%$ de sus lugares de trabajo tienen políticas que prohíben fumar en cualquier área de trabajo cerrado, sin embargo, $10.9 \%$ refirió haber estado expuesto al HTA en los últimos 30 días.

La exposición al HTA fue de $81.2 \%$ en bares y clubes nocturnos, de $29.6 \%$ en restaurantes, de $24.2 \%$ en el transporte público, de $17.0 \%$ en edificios del gobierno y de $4.3 \%$ en centros de atención médica.?

Uno de los grupos de población para el que resulta fundamental analizar la prevalencia de fumadores es el de los adolescentes, ya que, como hemos visto, en esa etapa se inicia el consumo de tabaco. La Encuesta de Tabaquismo en Jóvenes (ETJ) encontró que entre 8 y $15 \%$ de los estudiantes ha probado el cigarrillo antes de los 13 años de edad. No se debe perder de vista la relación entre el consumo de tabaco y las drogas ilegales. Medina-Mora y colaboradores encontraron que los mexicanos que empiezan a fumar antes de los 15 años de edad reportan mayor porcentaje de experimentación con drogas ilegales. ${ }^{6}$

La protección de la sociedad de los efectos nocivos del tabaco, se regula por leyes, normas, reglamentos y programas. El marco legal de la lucha antitabáquica en nuestro país comprende: el Reglamento sobre Consumo de Tabaco (julio de 2000) y de las Normas Oficiales Mexicanas 168-SSA11998 del Expediente Clínico (30 de septiembre 1999) y la NOM028-SSA2-1999 para la Prevención, Tratamiento y Control de las Adicciones, la Ley de protección a la salud de los no Fumadores en el distrito federal (29 de enero de 2004), la Ley General para el Control del Tabaco (30 de mayo de 2008) y el Reglamento de la Ley General para el Control del Tabaco (31 de mayo de 2009).9-12

Hasta 2008 la legislación para el control del tabaco se encontraba principalmente en la Ley General de Salud (LGS). Atendiendo a las recomendaciones del CMCT, México trabajó en una nueva iniciativa, la Ley General para el Control del Tabaco (LGCT), ${ }^{12}$ aprobada por las cámaras de Diputados y Senadores. Su reglamento fue publicado hasta el 31 de mayo de 2009 y entró en vigor hasta el 30 de junio de ese mismo año.

La mayoría de la legislación para el control del tabaco en México, con excepción de los impuestos, está bajo la LGCT. Ésta contempla seis apartados que establecen las atribuciones de la autoridad para hacer promoción, prevención y tratamiento a través del Programa Nacional para el Control del Tabaco; el consumo y la protección contra la exposición al HTA (Art. 26), también promueve la participación ciudadana (Art. 35) y establece las estrategias de vigilancia sanitaria, denuncia ciudadana y sanciones para el adecuado cumplimiento de la ley (Art. 36). ${ }^{9-12}$

En un análisis crítico, el СMCT, expresa algunas debilidades, una de ellas es que permite zonas exclusivamente para fumar en el interior de los espacios $100 \%$ libres de humo de tabaco. Aunque el reglamento especifica las características de dichos lugares, en la realidad es difícil implementar y vigilar su cumplimiento.

El 26 de febrero de 2008 la Ciudad de México aprobó la Ley de Protección a la Salud de los No Fumadores del Distrito Federal. ${ }^{9}$ 


\section{Enfermería Universitaria}

InNOVACIÓN para la PráCtica

Esta legislación que entró en vigor el 3 de abril de 2008 prohíbe fumar en todos los lugares públicos y de trabajo cerrados, incluyendo bares y restaurantes. Es una legislación integral que promueve los ambientes $100 \%$ libres de humo de tabaco, acorde con las recomendaciones del CMCT.

Para el éxito de la lucha contra el tabaquismo es indispensable contar con información y análisis permanentes, con objeto de orientar las medidas de prevención y control que fortalezcan la política fiscal del tabaco, eliminar la publicidad de los productos, crear ambientes y espacios libres de humo, evitar el acceso de los adolescentes al producto, suministrar información de manera permanente a la población sobre los daños, ofrecer opciones de tratamiento a los fumadores y propiciar la sustitución de cultivos.

En los últimos años nuestro país ha emprendido importantes acciones para el control del tabaco, los resultados descritos en las diferentes encuestas y estudios sirven en cierta medida para evaluar el cumplimiento de algunas de las acciones propuestas por el CMCT.

\section{Conclusiones}

La información que se ha obtenido en torno a la epidemia del tabaquismo en nuestro país revela la necesidad de garantizar el derecho fundamental a la salud en los jóvenes y a que disfruten de un entorno de vida y ambiente saludables.

El fomentar espacios $100 \%$ libres de humo de tabaco, que pro- mueve la reciente Ley de protección a la salud de los no fumadores en el Distrito Federal, reconoce este hecho, ${ }^{9-12}$ Las campañas para fomentar espacios libres de humo de tabaco deben centrarse en los aspectos positivos de no fumar, y deben estar particularmente orientadas a los jóvenes; así como considerar el reforzamiento de los siguientes elementos:

- Promover la educación sobre la salud durante todas las etapas del proceso educativo.

- Integrar a los jóvenes en la toma de decisiones. La estrategia de las organizaciones internacionales de promoción de la salud que considera que los jóvenes no son el problema sino la solución, nos invita a incorporar su propia

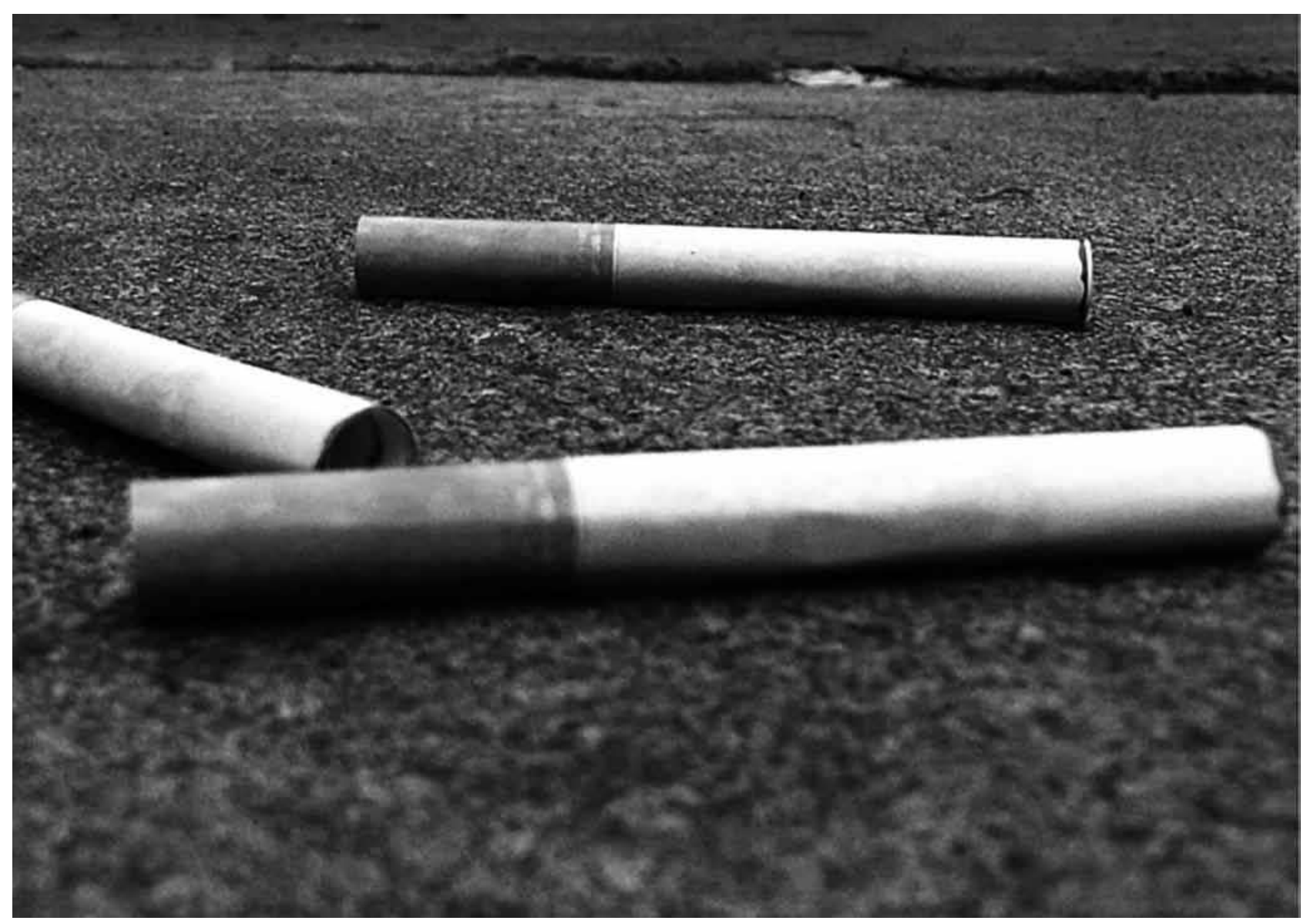


perspectiva para fomentar estilos de vida saludables.

- Desarrollar programas integrales sobre el significado de vida saludable, para ser integrados con carácter obligatorio en la educación formal, centrados en alternativas para la prevención y abandono del tabaquismo.

- Diseñar estrategias para dejar de fumar en los jóvenes, incluidas las terapias sustitutivas de la nicotina, que deberán ofrecerse gratuitamente y tener cobertura universal.

- Garantizar la prohibición de cualquier forma de publicidad del tabaco.

- Prohibir el empleo de colores en las cajetillas de cigarrillos, como medio sutil e indirecto para publicitar los productos del tabaco.

- Reglamentar advertencias sobre los daños a la salud de los productos de tabaco, que ocupen al menos el $75 \%$ de la superficie de las cajetillas.

- Promover campañas antitabaco focalizadas en los efectos nocivos del tabaquismo.

- Fomentar la abstinencia de consumo de cigarrillos en los jóvenes, privilegiando los valores positivos de una vida sin tabaco.

Finalmente podemos establecer que toda persona tiene derecho a un ambiente saludable y $100 \%$ libre de humo de tabaco, tanto en lugares cerrados como en lugares públicos delimitados.

Por consiguiente, el principio rector de las prohibiciones de fumar debe ser evitar la exposición al humo de tabaco en el ambiente, con énfasis especial en las escuelas, así como en lugares cerrados y espacios públicos, en el lugar de trabajo y en el hogar.

Para alcanzar este objetivo, enfatizamos la necesidad de contar con una reglamentación que promueva una prohibición de fumar integral, que abarque lugares públicos, escuelas, hospitales, transporte público, guarderías y lugares de trabajo, incluyendo lugares de esparcimiento (especialmente, hoteles, bares y restaurantes).

La premisa esencial es que la mejor opción para alcanzar estos objetivos es la prohibición total de fumar. Por esta razón es preciso evaluar y vigilar cómo se aplica la legislación sobre la prohibición de fumar. Sin duda el mejor legado a los jóvenes es garantizar espacios 100\% libres de humo de tabaco.

\section{REFERENCIAS BIBLIOGRÁFICAS}

1. Organización Mundial de la Salud. MPOWER: Un plan de medidas para hacer retroceder la epidemia de tabaquismo. Suiza: Organización Mundial de la Salud; 2008.

2. Secretaría de Salud, Programa de Acción: Adicciones. Tabaquismo. México: Secretaria de Salud; 2001.

3. Organización Panamericana de la Salud. Convenio Marco Contra el Tabaco. Washington, D. C.: OPS; 2001.

4. Organización Panamericana de la Salud. Desarrollo de legislación para el control del tabaco: Modelo y guías. Washington, DC.: OPS; 2002.

5. Organización Panamericana de la Salud. Exposición al humo de tabaco ajeno en las Américas: una perspectiva de derechos humanos. Programa de Control del Tabaco, Evaluación y Gestión de Riesgos Área de Desarrollo Sostenible y
Salud Ambiental. Washington, D. C.: OPS; 2004.

6. Organización Panamericana de la Salud. Planificación estratégica para desarrollar un movimiento por el control del Tabaco. Washington, D.C. OPS; 2004.

7. Reynales-Shigematsu LM, ValdésSalgado R, Rodríguez-Bolaños R, Lazcano-Ponce E, Hernández-Ávila M. Encuesta de Tabaquismo en Jóvenes en México. Análisis descriptivo 2003, 2005, 2006, 2008. Cuernavaca, México: Instituto Nacional de Salud Pública; 2009.

8. Organización Panamericana de la Salud; Instituto Nacional de Salud Pública. Encuesta Global de Tabaquismo en Adultos. México 2009. Cuernavaca, México: Instituto Nacional de Salud Pública. Organización Panamericana de la Salud. 2010.

9. Asamblea Legislativa del Distrito Federal, IV Legislatura. Ley de protección a la salud de los no Fumadores en el Distrito Federal. México, D.F.: Gaceta Oficial del Distrito Federal; 29 de enero de 2004.

10. Secretaría de Salud, Decreto por el que se expide la Ley General para el Control del Tabaco; México: Diario Oficial; 30 de mayo de 2008.

11. Modificación a la Norma Oficial Mexicana NOM-028-SSA2-1999, Para la prevención, tratamiento y control de las adicciones, para quedar como Norma Oficial Mexicana NOM-028-SSA2-2009, Para la prevención, tratamiento y control de las adicciones. México.

12. Reglamento de la Ley General para el Control del Tabaco. México: Diario Oficial; 31 de mayo de 2009. 